\title{
Predicting the volume shrinkage while manufacturing the GTE turbine blades
}

\author{
Roman Vdovin ${ }^{1, *}$ \\ ${ }^{1}$ Samara University, 34, Moskovskoe sh., Samara, 443086 Russia
}

\begin{abstract}
The process analysis of manufacturing the castings of turbine moving blades demonstrated that approximately $5 \%$ of the blade blanks from a lot are rejected to a considerable geometric distortion of the blade airfoil. The turbine moving blade casting manufactured by the casting method with directed crystallizing may differ in its geometric and dimensional-accuracy parameters from the design model. The casting geometry varies as a result of high temperature and structural shrinkage deformations which are manifested as the hindered volumetric shrinkage and contraction during crystallizing and upon the casting knockout from the ceramic mould. High-temperature deformations may result in contraction of the casting mould and ceramic core shaping the inner blade cooling channels. As a result, to obtain the blade of the geometric form as set by the designer, it becomes necessary to predict the stress-deformed state of the moving blade casting in order to consider the volumetric shrinkage and deformation in advance. Therefore, predicting and considering the total volumetric shrinkage during the manufacture of the moving blade castings ensuring the minimum contraction of the process system "ceramic mould - casting - ceramic core" is a relevant problem for the modern blank production.
\end{abstract}

\section{Introduction}

Improving the service life and reliability of gas turbine engines and units is presently conditioned by the necessity to increase the working temperature at the turbine inlet at simultaneous additional dynamic and vibration loads. First of all, this refers to the turbine blades which operate under the strength load and corrosion failure conditions during the interaction with aggressive gas flows for a long period of time. Therefore, the main efforts should be also aimed, among others, to improving the production technologies for the manufacture of the main engine parts such as blades [1-4].

For the modern thermostressed GTE, the above complex profile parts are manufactured by monocrystalline casting in multilayer shell forms with a subsequent machining of the blade root area and the shroud platform.

An important problem of technological preparation of turbine blade manufacturing is obtaining the faultless blanks. The nomenclature of the existing defects is wide and depends on a large number of factors. Within this work, the main reasons affecting the formation of

\footnotetext{
* Corresponding author: vdovin.ssau@gmail.com
} 
contraction defects and stressed condition in the blade castings as well as the methods for counteracting and predicting the behaviour of these deviations, will be considered [5].

\section{Study of the metal hardening process model}

The technology for manufacturing the ceramic refractory mould at the directionallysolidified casting is such that the ceramic mould has a layered structure, the thickness of each layer is $0.9-1.0 \mathrm{~mm}$ [6]. Therefore, each layer represents an isotrope material and, in general, may have thermophysical and physical-mechanical properties, which differ from other layers. Each layer consisting of fine sand is rigidly bound with the subsequent layer with the help of a binding material. Therefore, the ceramic mould is heterogeneous in general.

To eliminate cracking of the ceramic mould in casting and increasing the alloy flowability, the ceramic mould is heated to a particular temperature and only then it is filled with liquid metal.

During casting the molten metal in the ceramic mould a thermal shock occurs at the contact boundary. The form is heated extremely irregularly due to low value of the thermal conductivity factor of ceramics. The heated metal close to the contact boundary at the internal surface of the mould expands while compressing the zones not yet warmed up. Therefore the non-steady stress-deformed state of material develops, which corresponds to the time-changing contact boundary between the metal and mould.

At the initial moment of time, a two-component system is formed: liquid metal and ceramic mould (fig. $1 a$ ).

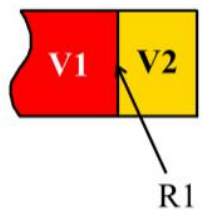

$a$

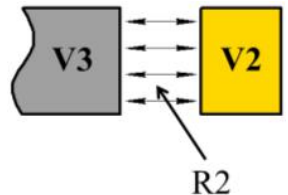

$b$

Fig. 1. Diagram of boundary conditions.

$a$ - liquid metal - ceramic mould contact zone; $b$ - crystallized metal - ceramic mould contact zone. V1 - liquid metal; V2 - ceramic mould; V3 - crystallized metal; R1 - contact boundary of liquid metal and ceramic mould; R2 - the gap between the crystallized metal and ceramic mould.

The crystallization process is initiated upon filling of the ceramic mould inner cavity with the molten metal. Moreover, the effect of the latent heat of metal crystallization is added to the thermal shock. The phase boundary appears, which is directed towards the casting centre in the course of time.

At this stage, the two-component system turns into a three-component one (fig. $1 \mathrm{~b}$ ): liquid metal - area 1, crystallized metal - area 2, ceramic mould - area 3 (fig. 2).

According to [7], at the non-uniform heating conditioned by the external heat source, the temperature field is practically not dependant on small deformations caused by it - the unrelated quasistatic problem setting. In this case, the thermal conductivity equation, according to [7] is written as (1).

$$
\nabla^{2} \theta-\frac{1}{a} \cdot \dot{\theta}+\frac{q_{m} \cdot \rho}{\lambda}=0
$$


where $\nabla^{2}=\frac{\partial^{2}}{\partial x_{1}^{2}}+\frac{\partial^{2}}{\partial x_{2}^{2}}+\frac{\partial^{2}}{\partial x_{3}^{2}}-$ Laplasian operator, $\theta$ - temperature, $\dot{\theta}=\frac{\partial \theta}{\partial \tau}, \tau-$ time, $a=\frac{\lambda}{C \cdot \rho}-$ temperature conductivity factor, $\lambda-$ thermal conductivity factor, $C-$ mass specific heat, $\rho$ - density. The value $q_{m}$ in the equation (1) represents the amount of heat generated per unit time in the volume of the substance unitary by mass.

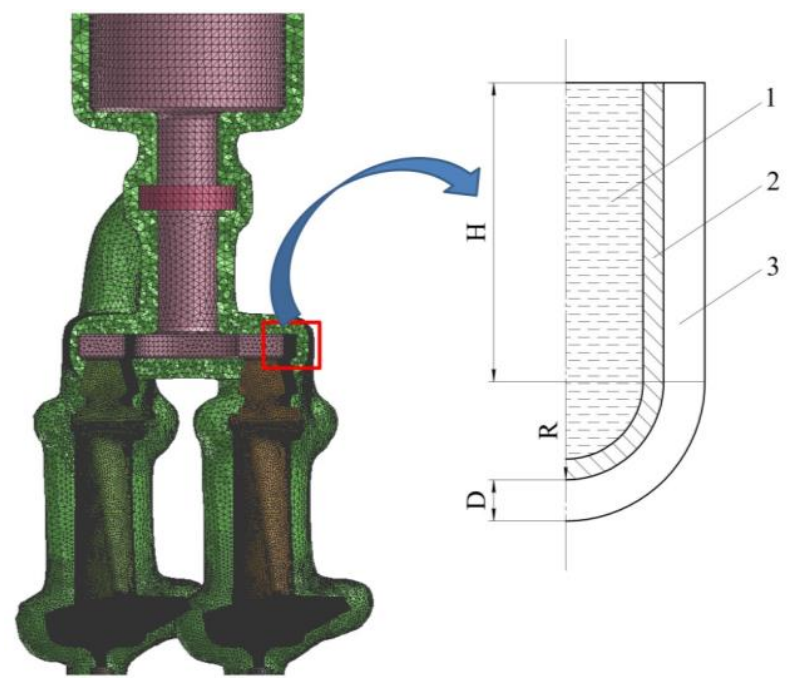

Fig. 2. Stress deformed state problem statement.

In this setting, the hidden crystallization heat generated during the molten metal hardening is considered by the compensation member $\left(q_{m} \cdot \rho\right) / \lambda$, which is different from zero for such zones of the area 1 (figure 1) which are in the two-phase state at the current moment of time (have the temperature between the liquidus and solidus temperature).

\section{Digital simulation of the shrinkage process}

During crystallization of the molten metal, its density increases, the casting gets shrinked and detaches from the ceramic mould. The analysis of literary sources and the production experience demonstrated that in the general case with the arbitrary shape and size of the casting such detachment takes place along the casting surface in a discrete way and is of a probabilistic nature $[8,9]$. However, only the presence of a considerable gas gap changes the contact heat exchange conditions.

Fig. 3 shows the behaviour of volumetric shrinkage of a block of castings of the turbine moving blades after crystallization. The initial casting geometry is designated in grey, the casting after shrinkage is shown in red. For the better visualisation, the shrinkage direction is shown with arrows. It should be noted that using the optimal process modes of the blade casting process enabled obtaining the uniform contraction of the moving blade casting [10, 11]. The computer simulation results demonstrate that the shrinkage is commonly nonuniform, however the following behaviour is revealed - the turbine moving blade casting may conditionally be divided into three areas, each of which is characterized by the uniform change of geometry during the thermal shrinkage [12]. Namely: shrinkage of the root area is $1.2-1.3 \%$; the casting airfoil shrinkage varies within $0.5-0.6 \%$; shrinkage of the upper shroud of the casting with the edges, due to thin walls of the blade structural elements, is 
$0.2-0.3 \%$. A series of numerical experiments demonstrated that the volume shrinkage of each of the blade casting areas is uniform and the shrinkage value varies within the mentioned boundaries.

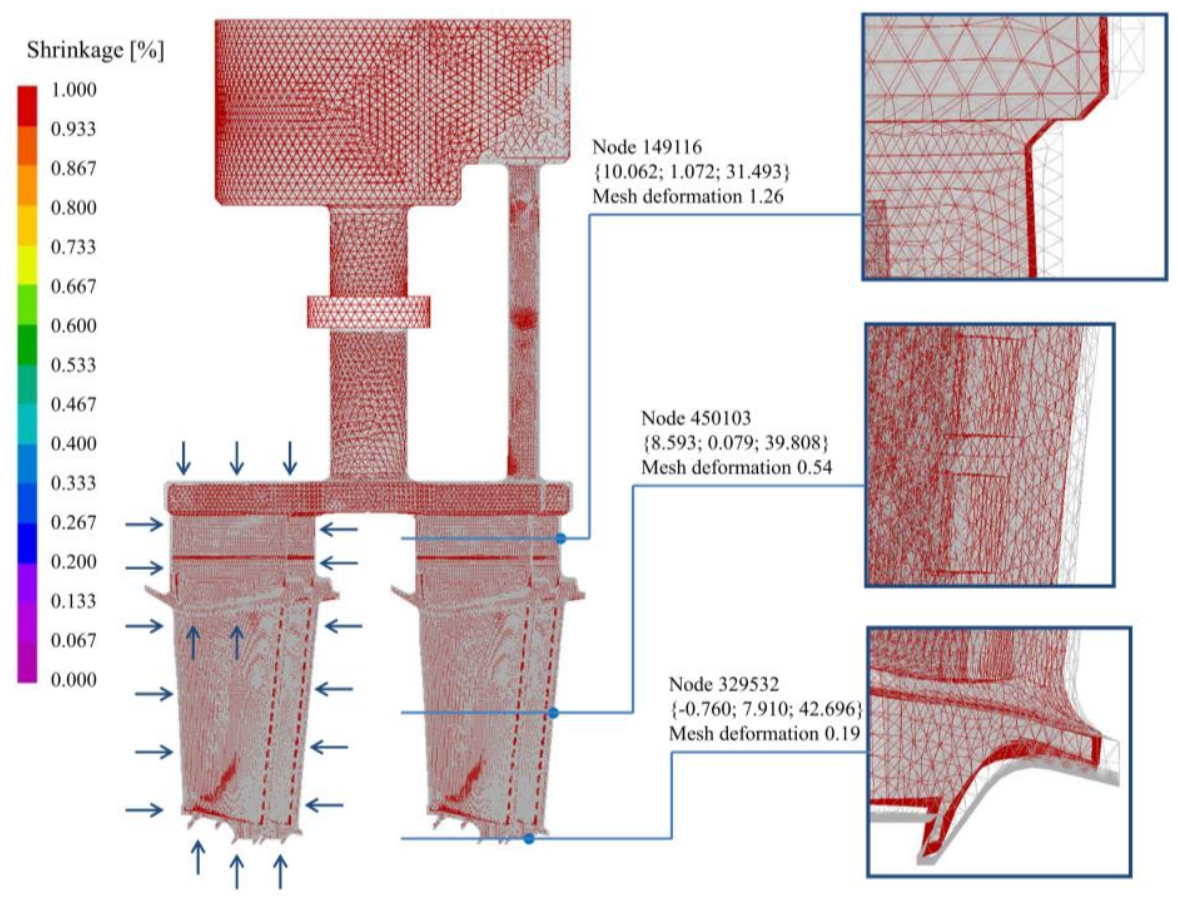

Fig. 3. The nature of the volume shrinkage of the casting block.

\section{Discussions}

The stability of simulation results was noted to be reached due to the selection of optimal modes of the directed crystallization modes, which were experimentally confirmed by a series of the experimental smelts. The results of the real castings showed that the amount of rejects in the blanks was managed to be reduced by approximately $5 \%$ due to their geometric failure. In addition, the uniformly changing geometry has a number of advantages during the shrinkage,

First of all, due to the uniform distribution of the tolerance in the blade blank, the need of local (point) polishing of the blade airfoil in the gauge device during preparation of the technological bases for the blade root machining becomes eliminated. Therefore, in the GTE turbine impeller, the position of the airfoil with respect to the gas approach flow will be constant and uniform for all the blades which will have a positive impact on the gas dynamic characteristics of the engine.

Secondly, stably varying geometry of the moving blade blank allows reducing the value of the embedded tolerance for the blade root machining and reducing material costs [13, 14].

\section{Conclusions}

The developed digital model of the metal crystallization process predicting the nature and localization of the volumetric shrinkage allowed to decrease the amount of rejects 
connected with the geometric inconsistency of the turbine blade blanks and the longitudinally oriented and monocrystalline structure totally by $5 \%$. Due to stability of the obtained geometric dimensions of the airfoil and wall thickness of the suction and pressure side in case of the cooled blades it becomes possible to perform the ultrasonic inspection of the blade wall thickness on a random basis (fig. 4).
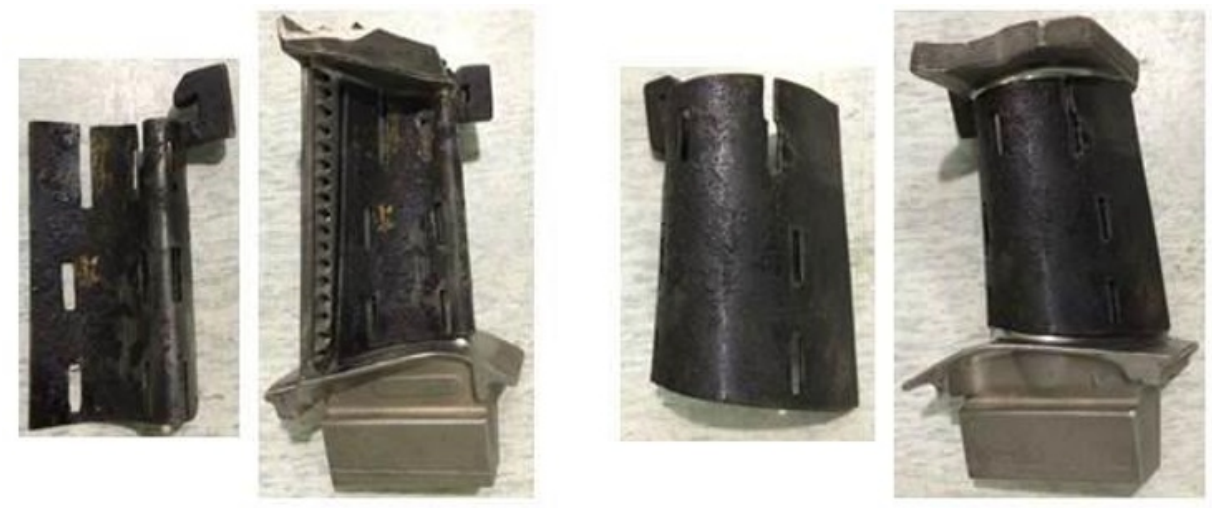

Fig. 4. The overlay templates for the ultrasonic inspection of the GTE turbine moving blade wall thickness.

This work was performed within the grant of the President of the Russian Federation for governmental support of young Russian Scientists - Candidates of Sciences (the competition - MK 2019).

\section{References}

1. N. Takeshi, Development of CMC turbine parts for aero engines, Engineering Review, v. 47(1), pp. 29-32 (2014)

2. E.M. Dobryshkina, A.V. Balyakin, R.A. Vdovin, V.P. Alekseev, Rapid Prototyping Technology for Manufacturing GTE Turbine Blades, IOP Conference Series: Materials Science and Engineering, v. 327(2), 8 (2018)

3. R.A. Vdovin, V.G. Smelov, Design and optimization of the micro-engine turbine rotor manufacturing using the rapid prototyping technology, IOP Conference Series: Materials Science and Engineering, v. 177(1), 6 (2017)

4. A.V. Balyakin, K.A. Bezsonov, M.V. Nekhoroshev, A.P. Shulepov, Developing parametric models for the assembly of machine fixtures for virtual multiaxial CNC machining centers, IOP Conference Series: Materials Science and Engineering, v. 302, $6(2018)$

5. R.A. Vdovin, V.G. Smelov, Research and optimization of the technological process of manufacturing a GTE blades using computer-aided design, IOP Conference Series: Materials Science and Engineering, v. 156(1), 7 (2016)

6. D.P. Szeliga, Modeling of directional solidification of columnar grain structure in CMSX-4 nickel-based superalloy, Materials Engineering and Performance, v. 23(3), pp. 1088-1095 (2014)

7. G.M. Sevastyanov, A.M. Sevastyanov, V.I. Odinokov, On a single boundary-value problem of heat conduction in a system with phase transitions in a shell form, Math modeling, v. 25(3), pp. 119-133 (2013) 
8. A.I. Khaimovich, A.V. Balaykin, A.I. Kondratiev, Methodology of rheological material properties phenoinenological modeling at high speed cutting by reverse analysis, Research Journal of Applied Sciences, v. 9 (11), pp. 753-760 (2014)

9. Y.Z Zhou, A.Volek, N.R. Green, Mechanism of competitive grain growth in directional solidification of a nickel-base superalloy, The Minerals, Metals \& Materials Society, v. 56(1), pp. 2631-2637 (2008)

10. R.A. Vdovin, V.G. Smelov, V.S. Sufiiarov, E.V. Borisov, Designing of the digital casting process for the gas turbine engine blades with a single-crystal structure, IOP Conference Series: Materials Science and Engineering, v. 441(1), 9 (2018)

11. R.A. Vdovin, Designing a duplicate model of the GTE turbine blade casting process, IOP Conference Series: Materials Science and Engineering, IOP Publishing Ltd (to be published)

12. D.P. Szeliga, The unidirectional crystallization of metals and alloys (turbine blades), Rzeszow University of Technology, v. 1, 45 (2015)

13. V.A. Pechenin, M.A. Bolotov, N.V. Rusanov, Method of evaluation of profile form and shaped surfaces with application of wavelets, Research Journal of Applied Sciences, v. 9(11), pp. 820-824 (2014)

14. V.A. Pechenin, M.A. Bolotov, N.V. Rusanov, Uncertainties in measuring the compressor-blade profile in a gas-turbine engine, Russian Engineering Research, v. 36(12), pp. 1058-1065 (2016) 